\title{
Publisher's Note: Attosecond-magnetic-field-pulse generation by electronic currents in bichromatic circularly polarized UV laser fields [Phys. Rev. A 92, 063401 (2015)]
}

Kai-Jun Yuan and André D. Bandrauk

(Received 8 March 2016; published 18 March 2016)

DOI: 10.1103/PhysRevA.93.039907

This paper was published online on 2 December 2015 with a typographical error in the label of panel (a) in Figs. 2 and 4. The label of panel (a) in Figs. 2 and 4 should read as "co-rotating." The figures have been corrected as of 3 March 2016. The figures are incorrect in the printed version of the journal, therefore, for the benefit of the print readership, the figures are replicated below.

(a) co-rotating

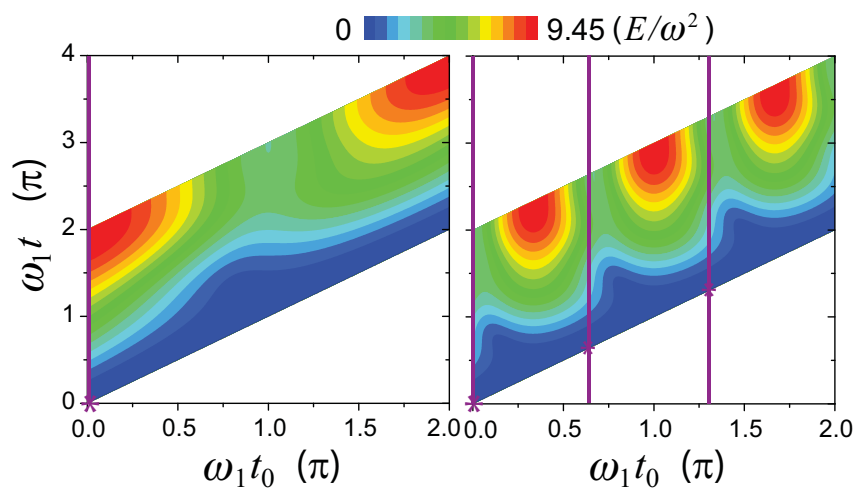

FIG. 2. Classical electron trajectories $r(t)=\sqrt{x^{2}(t)+y^{2}(t)}$ as functions of $\omega_{1} t_{0}$ and $\omega_{1} t$ by the bichromatic circularly polarized pulses for the (a) co- and (b) counter-rotating cases with CEPs $\phi_{1}=\phi_{2}=0$. Purple lines $(*)$ indicate the initial ionization time $t_{0}$ at maxima of electric fields; cf Fig. 1. (a) co-rotating

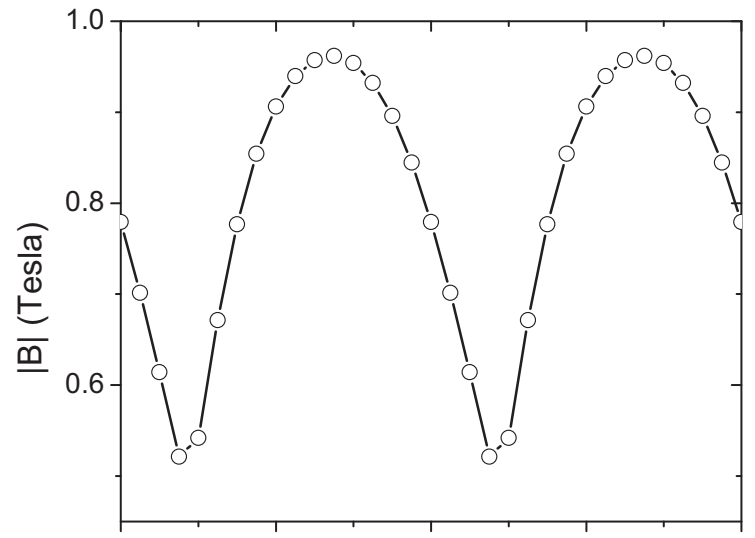

(b) counter-rotating

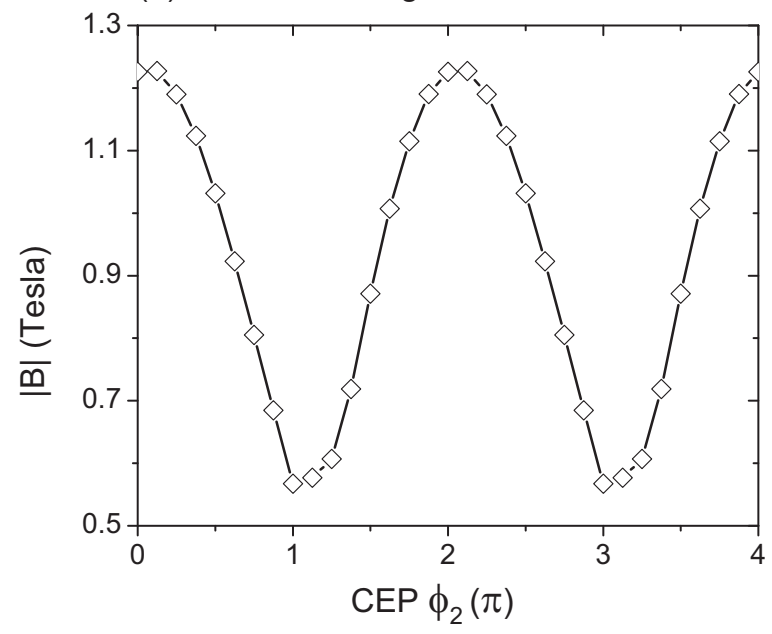

FIG. 4. Dependence of induced magnetic fields $|B|$ at the molecular center $O$ on the phases $\phi_{2}$ by (a) co-rotating and (b) counter-rotating bichromatic circularly polarized attosecond UV pulses for $x$ aligned $\mathrm{H}_{2}{ }^{+}$. The pulse wavelengths $\lambda_{1}=70 \mathrm{~nm}$ and $\lambda_{2}=35 \mathrm{~nm}$, duration $T=5 \tau_{1}=10 \tau_{2}(580$ as FWHM $)$, and intensity $I=1 \times 10^{16} \mathrm{~W} / \mathrm{cm}^{2}$. The CEP $\phi_{1}$ is always fixed at 0 . 